\title{
René Maran, une approche par les archives
}

\section{Xavier Luce et Claire Riffard}

\section{OpenEdition \\ Journals}

Édition électronique

URL : https://journals.openedition.org/coma/7798

DOI : 10.4000/coma.7798

ISSN : 2275-1742

\section{Éditeur}

Institut des textes \& manuscrits modernes (ITEM)

\section{Référence électronique}

Xavier Luce et Claire Riffard, «René Maran, une approche par les archives », Continents manuscrits [En ligne], 17 | 2021, mis en ligne le 15 octobre 2021, consulté le 09 janvier 2022. URL : http:// journals.openedition.org/coma/7798; DOI : https://doi.org/10.4000/coma.7798

Ce document a été généré automatiquement le 9 janvier 2022.

\section{(c) (i) () $\Theta$}

Continents manuscrits - Génétique des textes littéraires - Afrique, Caraîbe, dispora est mis à disposition selon les termes de la licence Creative Commons Attribution - Pas d'Utilisation Commerciale - Pas de Modification 4.0 International. 


\title{
René Maran, une approche par les archives
}

\author{
Xavier Luce et Claire Riffard
}

1 L'équipe Manuscrits francophones de l'ITEM a ouvert en 2020 un chantier de recherche autour des archives de René Maran. Plusieurs raisons motivaient ce choix. L'effet Goncourt, bien sûr, puisque 2021 est l'année du centenaire du Goncourt 1921 attribué à Batouala, véritable roman nègre qui fit connaître René Maran à travers le monde. L'enthousiasme de Bernard Michel ensuite, petit-fils de l'écrivain, qui souhaitait un regain des études maraniennes. Il met depuis tout en œuvre pour inciter, d'une part, la communauté des chercheurs à relire et étudier à nouveaux frais les textes de son grand-père ; d'autre part, pour que les institutions françaises valorisent son œuvre et l'inscrivent dans le patrimoine national. L'accès aux manuscrits enfin, clef de voûte pour une approche génétique telle qu'elle se pratique à l'ITEM. Or les archives de René Maran se révèlent d'une très grande richesse.

2 Nous allons commencer par les présenter, puis nous pointerons quelques pistes surgies de cette entreprise collective, toute récente et fragile encore, de connaissance de l'œuvre par les manuscrits, avant d'évoquer rapidement l'actualité maranienne de cette année du centenaire.

\section{Les archives René Maran}

René Maran conservait toutes les traces de genèse, y compris les notes préparatoires et les plans hâtivement griffonnés de ses futurs essais. Après sa mort le 9 mai 1960, ces brouillons ont été préservés de la destruction avec le même soin méticuleux. Sa veuve Camille, qui a continué d'habiter square Delambre à Paris, au deuxième étage d'une maison pleine de sa mémoire, a rangé à son exemple dans des chemises et des enveloppes les documents restés épars jusqu'alors.

4 Le meuble-bibliothèque $\mathrm{du}$ salon, particulièrement, contenait de très nombreux volumes ayant appartenu à son mari, sans doute près de 10000 ouvrages. Cet appétit de Maran pour les livres est attesté par ses amis, au premier chef le poète Léon Bocquet, 
éditeur de son premier recueil de poésie, La Maison du bonheur (1909): la littérature était, rapporte-t-il, « comme son vice élégant, son péché d'habitude, sa religion unique, la nécessité et l'aliment de son cerveau et de son âme ${ }^{1}$ ». " Impressionnante est, en effet, la culture livresque de Maran ", rappelle Sylvie Brodziak :

Loin de la France, patrie de sa formation intellectuelle, il a transporté et dévoré presque tous les livres, Virgile, Marc-Aurèle, Gide, Suarès, La Fontaine, Rutebeuf, Henri de Régnier, Renan, Albert Samain, Adam de la Halle, Charles d'Orléans, Villon, Rabelais, Malherbe, Louis Ménard, Bergson, Anatole France, Tagore... Il a feuilleté presque toutes les revues de son époque: Le Mercure de France, La Revue bleue, La Nouvelle Revue française, L'Amitié de France, La Vie, La Revue des deux mondes, Les Belles-Lettres...2

5 Nous connaissons avec exactitude le contenu de la bibliothèque de l'écrivain telle qu'il l'a laissée à son décès, car Camille Maran a pris le soin d'établir entre 1960 et 1967 une liste de ces volumes, à des fins de donation ou, nécessité faisant loi, dans la perspective de les vendre. L'étude de cet inventaire permettra de renforcer les études ayant trait à la formation de Maran ainsi qu'à l'importance des lectures dans la construction et l'évolution de ses positions politiques et intellectuelles. L'article de Roseline Garcia Ballester dans le présent numéro s'appuie substantiellement sur sa connaissance de la bibliothèque de l'écrivain.

\section{Archives de l'université Cheikh Anta Diop de Dakar}

6 En 1965 Camille Maran a fait en témoignage d'amitié une donation considérable à la République du Sénégal, dont le Président de l'époque, Léopold Sédar Senghor, était un ami de longue date de son mari. Ce don concerne de nombreux volumes de la bibliothèque de l'écrivain, mais aussi une bonne partie de ses archives. On y trouve plusieurs manuscrits et tapuscrits d'œuvres littéraires, dont un manuscrit du récit Un Homme pareil aux autres (Ms 15, intitulé «Un Homme comme un autre»), et une abondance de textes critiques de René Maran, conservés soit sous la forme de brouillons manuscrits ou dactylographiés, soit de coupures de presse. Une grande partie de ce fonds intitulé «Hommage de Madame Camille Maran à la République du Sénégal » est numérisée et accessible en ligne ${ }^{3}$.

\section{Archives familiales}

7 Au moment de verser en 1965 à la République du Sénégal une part conséquente de la bibliothèque et des archives de son défunt mari, Camille Maran a conservé par devers elle, pour des raisons affectives, certains volumes et certains documents - elle y porte l'annotation « pour moi ».

8 Après sa disparition en 1977, puis celle de sa fille adoptive, Paulette Cernard-Michel, en 2015, ce sont les enfants de Paulette, Bernard Michel et sa sœur Françoise, qui deviennent dépositaires de ces documents. Bernard Michel n'a pas connu son grandpère mais a eu une relation de grande proximité avec sa grand-mère.

La générosité de Bernard Michel a permis au groupe Maran, avec l'aide de trois étudiantes de Master de l'université Paris 8: Melissa Sidibe, Romane Oddon et Kéa Nanou, de dresser en janvier 2021 un inventaire précis des manuscrits et autres documents conservés dans les archives familiales. 
10 Le fonds, d'une exceptionnelle richesse, est constitué de deux gros cartons d'archives. Le premier contient une série de brouillons concernant Les Pionniers de l'empire (à la fois esquisses manuscrites, notes bibliographiques et " tapuscrits ", c'est-à-dire textes tapés à la machine, qui sont vraisemblablement des mises au net en vue d'une transmission à l'éditeur), et un dossier particulièrement volumineux concernant le dernier ouvrage publié par René Maran, Bertrand Du Guesclin. Dans le second carton, nous trouvons plusieurs états du Petit Roi de Chimérie, des documents très riches sur Bêtes de la brousse (notamment un feuillet portant des esquisses de plan de l'ouvrage, avec ratures et repentirs, deux exemplaires dactylographiés et annotés à la main, chacun avec des annotations différentes, de «Bassaragba le rhinocéros», idem pour «Mbala l'éléphant », un manuscrit de "Boum et Dog », plusieurs tapuscrits annotés de Bacouya le cynocéphale). À mentionner également, un dossier très riche sur Un homme pareil aux autres (sous le titre de Journal sans date - documents manuscrits et tapuscrits), et enfin un tapuscrit de Batouala.

\section{Bibliothèque municipale de Bordeaux}

11 Le réseau des bibliothèques municipales de Bordeaux possède de nombreuses lettres de René Maran écrites entre 1912 et 1960, principalement adressées à Charles Barailley, ainsi que trois cahiers intitulés "Batouala le mokoundji» (BX19104), «La Vie intérieure » (BX19105) et « Le Chemin de la solitude» (BX19106). Il s'agit de manuscrits annotés de la main de l'auteur. Ces archives appartenaient initialement au fonds Auguste Pujolle. Mais à compter de cette année, un fonds René Maran a été créé qui accueillera deux nouvelles acquisitions: une lettre autographe de René Maran à Jacques Boulenger postée de Fort-Archambault en date du 29 octobre 1921; une autre adressée à son condisciple Félix Éboué, postée à Paris et datée du 21 avril 1929. Il y est question du recueil Le Visage calme, du Petit Roi de Chimérie et du Roman d'un nègre (Un homme pareil aux autres) et de recommander Jacques Boulenger auprès de ses amis éditeurs Georges et Antoinette Mornay. À son "cher Félix ", l'écrivain raconte sa vie recluse, liée à sa condition d'«homme de couleur» et à son intransigeance de caractère : il revient ainsi sur son refus de «mercantiliser » son prix Goncourt dont il tâche, chaque jour, d'être digne.

12 L'axe Écritures contemporaines Caraïbe-Amazonie au sein de la bibliothèque numérique Manioc (universités des Antilles et de Guyane) proposera en 2022 un accès à certains de ces manuscrits.

\section{Archives nationales d'Outre-mer}

C'est à Aix-en-Provence, aux Archives nationales d'Outre-mer, que se trouve le dossier administratif de René Maran ainsi que celui de son père, le Guyanais Herménégilde Maran. Ce dernier, «appelé par [Savorgnan de] Brazza » au Gabon dès 1891 après quatre années en Martinique (1887-1891), avait fait tout le reste de sa carrière dans l'administration coloniale en Afrique, commençant au rang de commis pour finir gouverneur par intérim en Oubangui-Chari. René Maran marchait donc sur les traces de son père, lorsqu'il le rejoignit à Bangui en 1910, et se heurta comme lui à toutes sortes de difficultés dans l'exercice de ses diverses fonctions. Dans plusieurs instances, ces difficultés étaient liées au racisme de certains collègues fonctionnaires et de 
certains colons d'Afrique équatoriale française (AEF) ou du Congo belge (par lequel il fallait transiter pour rejoindre les postes au nord du fleuve).

Les pièces constituant le dossier de René Maran permettent de suivre la détérioration de ses relations professionnelles, déjà mauvaises avant 1918, détérioration grandement accélérée par le «scandale » de Batouala prix Goncourt en 1921. À telle enseigne qu'il fut conduit à solliciter, en haut lieu, une protection et une dérogation pour assurer son retour en France métropolitaine en 1923 : Maran estimait, en effet, que sa vie était en péril. Le retentissement médiatique provoqué par l'obtention du Goncourt risquait d'attirer l'attention de l'opinion publique sur les «deux rapports circonstanciés, appuyés de copies annexes de pièces officielles» dans lesquels Maran relatait «les iniquités et les crimes commis en Afrique équatoriale française par plusieurs fonctionnaires ${ }^{4}$ ». Ces rapports avaient été remis par l'écrivain en 1920 au ministère des Colonies, probablement lors du séjour à Paris au cours duquel il rencontra Henri de Régnier, directeur de la collection «Roman littéraire» chez Albin Michel et «tuteur » de Batouala (1921) avec Jacques Boulenger, rédacteur en chef de L'Opinion où une parution du roman en feuilleton avait été prévue.

15 À ces pièces administratives conservées dans la série Dossiers de personnel (EE II), s'ajoutent celles rassemblées par l'administrateur colonial André Fraisse ${ }^{5}$ lors de son gouvernorat en Afrique noire dans les années 1950. Celles-ci se trouvent dans la série des archives privées d'Outremer (8 APOM). Une note manuscrite, jointe à une lettre de René Maran, en présente l'intérêt : «Ces documents permettent d'expliquer en partie la révolte de René Maran contre l'homme blanc.»Et, plus loin, il est question de "ces feuilles, rongées par les termites, que j'ai pu sauver... ». Reçu au domicile du couple Maran, Fraisse note : «Je comprends, cette photo d'Hô Chi Minh dédicacée, sur le mur, - et son refus de préfacer mes contes sur l'Indochine ${ }^{6}$."

La carrière de Maran en tant que commis des services civils aux affaires indigènes constitue le contexte d'écriture de son œuvre romanesque. Celle-ci est à l'origine de son engagement à la croisée d'associations et de réseaux militants pour la défense des populations indigènes telle que la Ligue universelle de défense de la race nègre (LUDRN), à laquelle succèdera le Comité de défense de la race nègre (CDRN). À ce titre, fort de sa renommée littéraire, sa diatribe contre la Civilisation et sa fréquentation des réseaux communistes lui vaut d'être l'objet d'une surveillance attentive par les services de renseignement. C'est ainsi que son nom figure dans plusieurs documents de la série Service de liaison avec les originaires des territoires français d'Outre-mer (SLOTFOM) au point d'avoir été classé dans le bord communiste des membres du CDRN à la suite d'un entretien accordé à L'Humanité dans les mois précédant la parution de Djouma, chien de brousse (1927). Il s'agit de notes d'agents par lesquelles sont transmis les propos qu'aurait tenus l'écrivain lors de séances du CDRN entre 1924 et 1929, essentiellement. Ces notes s'avèrent précieuses dans la mesure où elles permettent de situer l'écrivain dans son époque afin d'apprécier la portée de son militantisme assimilationniste et la fonction sociale et politique qu'il assigne à son « métier d'écrivain français ».

17 L'ensemble de ces fonds d'archives, on l'aura compris, est conséquent et d'une grande richesse au plan génétique. À ceux-là, encore faut-il ajouter les documents épars dans les fonds de la BnF, telle que celui de Maurice Barrès (NAF 28210) et Marcel Martinet (NAF 28352), de l'INA et de bien d'autres institutions, en France, mais pas seulement: aux États-Unis, la bibliothèque Arthur Schomburg et la bibliothèque Moorland de l'Université Howard conservent d'autres tapuscrits et lettres de l'écrivain. 


\section{Pistes pour une connaissance de l'œurre par les archives}

\section{Brouillons}

18 L'accès aux brouillons des œuvres éditées permet de considérer le souci de perfection qui anime l'écrivain ainsi que le souligne Léon Bocquet dans la préface du Petit roi de Chimérie. Nous pouvons alors entrevoir la fabrique du texte, du moins dans la mesure de ce qui nous est parvenu. Dans le cas de Maran, les dossiers génétiques des œuvres sont inégalement riches. Certains livrent d'ores et déjà de nombreux éléments pour l'analyse génétique. Démonstration en est faite dans ce numéro à propos du dossier Un homme pareil aux autres, avec d'une part l'article de Roger Little sur la question du titre, et d'autre part les deux articles - complémentaires - rédigés par Tina Harpin et Laura Gauthier-Blasi sur ce récit d'inspiration autobiographique. D'autres ensembles sont surprenants: le dossier de genèse de Batouala, commenté ici par Charles Scheel, comprend par exemple un document singulier, tapuscrit aux allures de manuscrit, qui n'a pas encore livré tous ses secrets.

Certains documents tout à fait inconnus sont apparus à l'occasion de l'inventaire des archives Bernard Michel effectué en janvier 2021 par le groupe Maran de l'ITEM. Ainsi le fragment intitulé « Une amie », présenté ici par Roseline Garcia Ballester.

\section{Correspondance}

20 La correspondance d'un écrivain a toujours ceci de saisissant qu'elle nous permet l'accès aux dimensions biographique et génétique. D'une part, c'est une multitude d'informations, un fourmillement de scènes de vie et de détails anecdotiques, tantôt savoureux, qui ne font alors que renforcer notre sympathie à son égard; tantôt déconcertants, qui peuvent a contrario inspirer notre réprobation. Cet écrivain n'aurait-il donc été qu'un homme parmi les autres? De fait, les papiers d'un écrivain possèdent une force probante qui semble extérieure à la littérature ; c'est la dimension biographique. D'autre part, au détour d'une lettre, voilà que cet homme nous parle de son métier, qu'il annonce à tel ami un projet littéraire ou qu'il répond à un journaliste, à un lecteur, ou bien qu'il traite d'affaires contractuelles avec un éditeur; c'est la dimension génétique. Ces éléments permettent de cerner les contours de cette figure auctoriale embusquée dans l'œuvre, c'est-à-dire cette image projetée de l'écrivain.

21 Avec René Maran, une grande partie de la correspondance est particulièrement fascinante: alors qu'il est essentiellement connu comme l'auteur de Batouala, on découvre un poète ou, plutôt, un romancier qui se fait précéder de sa poésie. Ainsi, dans le but d'« inciter» Henri de Régnier «à jeter un coup d'œil sur [ses] pages oubanguiennes [de la colonie d'Oubangui-Chari] », le poème "Psyché » avait " précédé de peu l'envoi de la série d'eaux-fortes composant ce récit [Batouala] »'?

Cette correspondance évoque par ailleurs deux questions hautement controversées dans l'entre-deux-guerres : la colonisation française et l'assimilation. Leur articulation en tant qu'utopie fidèle aux idéaux républicains pose problème et conditionne la reconnaissance auctoriale dans le champ littéraire - celui de l'époque mais aussi celui d'aujourd'hui. Ainsi, lorsque Maran sera attaqué au sujet de Batouala, Léon Bocquet 
publiera un long plaidoyer nourri d'extraits des nombreuses lettres écrites par Maran depuis son poste d'agent colonial comme autant de témoignages de sa probité et de son patriotisme. Le texte de cette plaidoirie, légèrement modifié, sera repris pour servir de préface au deuxième roman publié de Maran, Le Petit roi de Chimérie (1924). Le cœur de son argumentaire a pour objet le racisme, qu'on désigne alors, d'un air entendu, comme un " préjugé de couleur ». Bocquet de s'exclamer, indigné : «Comme si cela importait le moins du monde au mérite et à l'intelligence ! " Et de préciser avoir ignoré que Maran fut un " homme de couleur " jusqu'à ce que l'intéressé ne le lui révélât, sur le ton d'un aveu, « avec l'air de s'excuser » note-t-il. De là une plus grande sympathie devait lier les deux hommes: «De nature tendre et sentimentale, lui qui n'avait pas toujours tiré toute joie d'avouer sa race, devint bientôt plus expansif. » L'écrivain s'épanche dans ces lettres écrites durant la gestation de son œuvre romanesque, à compter de fin 1909.

La collecte de ces ensembles de lettres, qui couvrent près d'un demi-siècle, permet de comprendre à quels réseaux intellectuels et littéraires il appartenait. Ses premiers correspondants, en dehors de Léon Bocquet et de Manoel Gahisto, ses conseillers littéraires, étaient naturellement ses amis de Bordeaux (Paul Culine, Louis Despaix, André Lafon, Charles Barailley...). C'est en vertu de ses liens d'amitié avec Léon Bocquet, mais aussi Louis Despaix, Phileas Lebesgue, André Lafon et Jean Balde pseudonyme de Jeanne Alleman, fiancée d'André Lafon -, au début de l'année 1917, que Maran initie une correspondance avec Maurice Barrès, le "plus grand des agitateurs d'énergie de la France qui se bat", en se présentant comme "nègre martiniquais ". Xavier Luce étudie ces lettres, peu nombreuses mais néanmoins importantes par leur contenu et leur contemporanéité de l'écriture de la préface de Batouala : s'y dessine son ambition auctoriale.

24 L'article de Claire Riffard permet de reconstituer en suivant les traces de leurs échanges épistolaires l'amitié au long cours que René Maran a nouée avec l'écrivain malgache Jean-Joseph Rabearivelo ; l'un et l'autre semblent mus par une même « froide colère ». Il est temps enfin de reconsidérer la relation concurentielle de René Maran avec André Gide, à la lumière notamment des correspondances entre Gide et l'administrateur colonial Marcel de Coppet, ce à quoi nous invite Nimrod.

Voici une liste non exhaustive de ses correspondants: Charles Barrailley, Maurice Barrès, Léon Bocquet, Jacques Boulenger, Gaston Chérau, Mercer Cook, Paul Culine, Lucien Descaves, Félix Eboué, André Fraisse, Manoel Gahisto, Charles Kunstler, Pierre Mac Orlan, Raoul de Malétable, Marcel Martinet, Albin Michel, Paul Léautaud, Alain Locke, Georges Henri Rivière, André Suarès, Jean Texcier, Daphné Trévor.

\section{Coupures de presse}

L'inventaire des textes critiques écrits par René Maran est à peine ébauché, mais la somme de ses contributions à la presse s'avère d'ores et déjà considérable et très éclectique. Cet ensemble livrera sans nul doute des éléments déterminants pour apprécier le positionnement politique de l'écrivain, ainsi que le formule Laure Demougin :

[...] il [Maran] apparaît comme un essayiste colonial pris entre deux positions et deux mondes, non pas défenseur de la colonisation, mais connaisseur de ses réalités et penseur de ses évolutions, souvent par le prisme littéraire. Jean-Pierre Dubarry pointe, à travers la présentation d'une coupure de presse tirée des Lettres françaises du 31 mars 1946, l'intérêt de Maran pour le pôle intellectuel 
indochinois, Yves Chemla sa curiosité pour les écrivains d'Haïti et Hervé Sanson la lecture très informée qu'il fait de son confrère algérien Mohammed Dib.

Quant à l'analyse du corpus critique produit sur Maran, et plus spécifiquement sur la réception de Batouala dans la France de 1921, elle est menée ici par Ferroudja Allouache, dans le prolongement de son ouvrage Archéologie du texte littéraire dit francophone: 1921-1970 (Classiques Garnier, 2018).

\section{Actualité maranienne}

L'année d'hommage a débuté en Martinique. La Collectivité territoriale de Martinique a organisé un colloque le 15 janvier 2021, puis le 22 mars un hommage qui associait les lycéens de l'île. Le 26 mars, le Printemps des poètes accordait grâce aux efforts de Martial Sinda une place importante à l'œuvre de René Maran. Le 28 mai, une journée d'étude était organisée à l'université Paris-VIII par Françoise Simasotchi et Ferroudja Allouache. En juin, un colloque avait lieu au Brésil, à l'université férédale Do Acre/UFAC de Rio Branco, sous la direction de Dennys Silva-Reis et de Danielle Grace. Le 26 septembre, une table-ronde autour de René Maran faisait salle comble au Salon du livre africain de Paris. La Collectivité territoriale de Guyane (CTG) organisait un colloque les vendredi 8 et samedi 9 octobre 2021. Buata Malela et Matthieu Renault, que nous remercions pour leur participation au séminaire René Maran et à l'élaboration de ce numéro, y proposaient des communications intitulées respectivement: "Maran, poète du sujet » et « René Maran ou les dilemmes de l'assimilation ». Le Salon de lecture du musée du quai Branly accueille samedi 16 octobre une séance ouverte à tous sur l'actualité de l'œuvre de René Maran. D'autres évènements sont prévus, que l'on peut consulter en suivant ce lien.

Si la célébration du centenaire du prix Goncourt 1921 suscite un important regain d'intérêt pour l'œuvre de René Maran, souvent réduite au seul roman primé, Batouala, véritable roman nègre, et à sa préface, il faut s'en réjouir car c'est l'œuvre d'un écrivain ayant marqué la période de l'entre-deux-guerres et joué un rôle de « mentor » pour la génération de Léopold Sédar Senghor et Aimé Césaire. Son discours préfaciel, comme l'a analysé Ferroudja Allouache dans sa thèse, présente un caractère inaugural pour l'étude de la réception et de la fabrication du texte littéraire "francophone». Le documentaire produit par Bérénice Médias Corp avec France Télévisions réalisé par Fabrice Gardel et Mathieu Weschler avec Alexia Klingler, René Maran, le premier Goncourt noir (2021), propose une reconstitution de cet évènement dans les lettres françaises à partir d'images d'archive habilement montées. On y entend aussi les académiciens Amin Maalouf et Dany Laferrière, l'écrivain Daniel Maximin et les professeurs Romuald Fonkoua et Françoise Simasotchi. Un podcast radiophonique réalisé par Alexia Klingler en prolongera le propos.

Les initiatives éditoriales ne sont pas en reste. Les éditions marseillaises du Typhon rééditent Un homme pareil aux autres, assorti d'une préface lumineuse du romancier Mbougar Sarr qui nous invite à plonger dans l'univers fictionnel de Maran, tandis que les éditions SCITEP republient le recueil de contes Bêtes de la brousse, depuis longtemps introuvable, avec une préface et des commentaires de Roger Little, directeur de la collection "Autrement mêmes" chez l'Harmattan dans laquelle il avait édité un ensemble de Nouvelles africaines et françaises : inédites ou inconnues (2018). Enfin, l'éditeur historique Albin Michel propose une nouvelle édition de Batouala - plus sobrement 
sous-titrée roman au lieu de véritable roman nègre et assortie d'une préface d'Amin Maalouf, auteur du Dérèglement du monde (Grasset, 2009).

\section{NOTES}

1. Léon BocQuet, « Préface », dans René MARAN, Le Petit Roi de Chimérie, Albin Michel, 1924.

2. Sylvie BRODZiaK, "Le Petit Roi de Chimérie de René Maran ou la littérature vigie de l'histoire », Présence africaine, $\mathrm{n}^{\circ}$ 187-188, 2013, p. 199.

3. http://bibnum.ucad.sn; rechercher par auteur et par titre d'ouvrage.

4. Cf. question écrite déposée par le député Achille René-Boisneuf lors de la séance de l'Assemblée nationale du 23 février 1922.

5. André FRAISSE (1909-1987), ancien administrateur de la France d'Outre-mer, en Indochine et en Afrique (1933-1960).

6. Voir dans ce même numéro le document commenté par Jean-Pierre Dubarry.

7. René MARAN, « Henri de Régnier », Vendémiaire, 15 avril 1936, p. 5.

\section{INDEX}

Keywords : René Maran, archives, colonization, prix Goncourt

Mots-clés : René Maran, archives, colonisation, prix Goncourt

\section{AUTEURS}

\section{XAVIER LUCE}

XAVIER LUCE est doctorant à l'Université Sorbonne au sein du Centre international d'études francophones (CIEF) et du Centre d'études de la langue et des littératures françaises (CELLF). Sa thèse, sous la direction de Romuald Fonkoua, porte sur Maryse Condé et sa critique. Il coordonne avec Claire Riffard au sein de l'ITEM le séminaire René Maran.

\section{CLAIRE RIFFARD}

CLAIRE RIFFARD est ingénieure de recherche à l'ITEM (CNRS-ENS). Depuis 2008 elle dirige l'équipe «Manuscrits francophones », qui étudie et édite les corpus littéraires majeurs d'Afrique et de la Caraïbe. Elle a coédité l'œuvre complète de Jean-Joseph Rabearivelo (t. I et II, CNRS Éditions, 2010-2012, 3000 p.) ainsi que les Poèmes de Sony Labou Tansi (CNRS Éditions, 2015, 1272 p.). 